\title{
The investigation of radionuclides distributions in beach sand by means of GIS techniques
}

\author{
Jacques Bezuidenhout ${ }^{1}$ \\ ${ }^{1}$ Faculty of Military Science, Stellenbosch University, South Africa, jab@ ma2.sun.ac.za
}

DOI: http://dx.doi.org/10.4314/sajg.v4i4.7

\begin{abstract}
A transportable and robust gamma ray detection system (GISPI) was employed to determine the concentrations of naturally occurring radionuclides on various beaches in and around Saldanha Bay, located on the West Coast of South Africa. Several mathematical techniques were applied to obtain GIS overlay that could be used to investigate various geophysical phenomenon. The results demonstrated direct relationships between radionuclide concentrations, grain size of beach sediment and the intensity of wave action. The element concentrations were used to make deductions on the origin of some of the beach sand. The article finally draws conclusions on coastal and beach formation processes, based on the results of the distribution of various radionuclides.
\end{abstract}

\section{Introduction}

The measuring of naturally occurring radioactive material is of great interest in various Earth Sciences like exploration, environmental monitoring and the classification of soil and rocks. Distinctive concentrations of these nuclides relate to geological and morphological features of specific locations. Igneous rock and regolith originating from such rock typically has higher concentrations of naturally occurring radionuclides than sedimentary rock. The natural and anthropogenic processes distinctly alter the concentrations and distribution of these nuclides [1]. The variation in these nuclides can therefore be utilised to investigate these processes and subsequently provide a better understanding of the underlining geophysical and human interactions.

Saldanha Bay consists of a complex of larger and smaller bays, all lined by beaches. The Atlantic Ocean waves are predominantly incident from the south-west [2,3], but the waves change direction in the various bays due to diffraction and reflection from the granite protrusions and peninsulas. Consequently beach deposits and sand varies from beach to beach. Saldanha Bay was substantially altered during the previous century, mainly by the construction of a breakwater and a long iron ore jetty. This significantly altered the wave intensities and patterns in various parts of the bay. As a result of this beach deposit patterns started to change, with several beaches showing signs of erosion. These structures furthermore had an impact on the content of the sand on the beaches and the accompanying intrinsic naturally occurring radionuclides concentrations of the sand. The variation of these naturally occurring radionuclides can therefore provide important information on geophysical changes.

The most significant naturally occurring radionuclides are uranium, thorium and potassium which are all of primordial origin [4]. The most abundant and transportable of these naturally occurring nuclides is potassium [5]. The nuclide ${ }^{40} \mathrm{~K}$, which is the 
only radionuclide in naturally occurring potassium, constitutes $0.01193 \%$ of the weight of potassium [6]. The ${ }^{40} \mathrm{~K}$ chain only emits a single gamma ray and this decay is utilized to obtain the concentration of potassium in natural material. The thorium $\left({ }^{232} \mathrm{Th}\right)$ and uranium $\left({ }^{238} \mathrm{U}\right)$ concentrations are typically determined from the decay of the daughter nuclides, which emits relatively high energy gamma rays. Natural uranium comprises ${ }^{238} \mathrm{U}(99.28 \%)$ and ${ }^{235} \mathrm{U}(0.71 \%)$, whereas natural thorium is totally constituted of ${ }^{232} \mathrm{Th}$.

Radionuclide surveys of these naturally occurring radionuclides were undertaken on three beaches in the area of Saldanha Bay. The following chapters deal with the method and results of these surveys and explore the possible reasons for variations in the concentrations of naturally occurring nuclides.

\section{Method}

\subsection{Measuring System}

The Gamma ray In Situ Portable Instrument (GISPI) was used to radio survey all the areas that were studied and the method was based on work done by Bezuidenhout [7]. The GISPI consists of a NaI(Tl) scintillation detector, a digital Multi Channel Analyser (MCA), a rugged tablet PC with an on-board GPS and real time analyses software that controls the whole system (see Figure 1). The $\mathrm{NaI}(\mathrm{Tl})$ detector $(7.62 \mathrm{~cm} \times 7.62 \mathrm{~cm}$ ) is coupled to the MCA and sealed in a padded case to protect the instruments form mechanical shock and dust. The scintiSPEC ${ }_{\circledast}$ MCA [8] that is produced by FLIR ${ }_{\circledast}$ has a USB connection that acts as the power source for operation and allows data transfer. A Trimble $_{\circledast}$ Yuma rugged tablet PC [9] with on-board GPS manages the systems and stores the data.

The system settings and spectrum acquisition were controlled by the winTMCA32 $\circledast$ software (with 1024 channels), that is also produced by FLIR ${ }_{\circledast}$. Program code was developed in the winTMCA32 software to analyse spectra and obtain geographical locations, as well as to export this data in GIS compatible format. The winTMCA32 code directly acquired the geographical position coordinated from the onboard GPS via a virtual communications port. The code also extracted the time corrected counts from the various energy windows and combined the radiation data with the positions data. The code finally stored all hardware settings, in situ spectra and the extracted results in files. The result files were read into, and interpreted using QGIS software.

\subsection{Calibrations}

Energy calibrations were performed before and after each period of measurement. The calibrations were done in the range from $0.2 \mathrm{MeV}$ to $2.7 \mathrm{MeV}$ using anthropogenic nuclides and natural environmental spectra. The following nuclides and associated gamma ray emissions were used for the energy calibration: ${ }^{214} \mathrm{~Pb}(351.3 \mathrm{keV}),{ }^{137} \mathrm{Cs}$ $(661.7 \mathrm{keV}),{ }^{60} \mathrm{Co}(1173.2 \mathrm{keV}, 1332.5 \mathrm{keV}),{ }^{40} \mathrm{~K}(1460.8 \mathrm{keV}),{ }^{214} \mathrm{Bi}(1764.5 \mathrm{keV})$ and ${ }^{208} \mathrm{Tl}(2614.5 \mathrm{keV})$. The symmetry assumptions and corrections described by McCay et al. [10] were adopted for the calibrations and surveys.

The efficiency calibration measurements of the system were done at standard radiation reference pads of the Nuclear Energy Corporation of South African (NECSA) according to the method described by Chiozzi and others $[11,12]$. These calibration spectra were used to extract efficiency their parameters for each of the nuclides according to the 
method described in paragraph on Measurements and Analyses. The nuclide concentrations were expressed in Becquerel per kilogram.

\subsection{Measurements and analyses}

The ${ }^{40} \mathrm{~K}$ emission is commonly very strong in natural spectra, due to the abundance of potassium in nature. An active stabilisation function in the winTMCA32 software was used to correct for energy drift, which results from temperature changes while the site is being surveyed. The $1460.8 \mathrm{keV}$ emission of ${ }^{40} \mathrm{~K}$ was chosen as centroid for stabilisation and the fine gain were automatically adjusted by the winTMCA32 software to correct for any drift from the peak.

The following procedure was followed for efficiency calibration. Three counting ROIs that were adapted were $1460.8 \mathrm{keV}, 1764.5 \mathrm{keV}$ and $2614.5 \mathrm{keV}$ and were denoted by $i$ equal to 1,2 , and 3, respectively. The calibration standards of potassium, uranium and thorium were indicated by $j$ equal to 1,2 and 3 , respectively. The net count rate $R_{i, j}$ in the $i$ th ROI of a calibration standard $j$ was then proportional to the activity $A_{n, j}$ of each investigated nuclide $n$. If $n=1,2$ and 3 denoted the ${ }^{40} \mathrm{~K},{ }^{238} \mathrm{U}$ and ${ }^{232} \mathrm{Th}$ nuclides, the net count rate were then express as

$$
R_{i, j}=\sum_{n=1}^{3} e_{i, n} A_{n, j}
$$

where $e_{i, n}$ gave the counting efficiency in the $i$ th ROI for the nuclide $n$. The net count rate in the ROI was given by

$$
R_{i, j}=\frac{N_{i, j}}{t_{j}}
$$

where $N_{i, j}$ was the counts in the $i$ th ROI for calibration standard $j, t_{j}$, was the counting time for standard $j$.

Eq. (1) is a linear system of $i \times n(3 \times 3)$ simultaneous equations. The counting efficiencies could be solved by relating the net count rates of the ROIs in the spectra of calibration standards with the concentration of the nuclides in those standards.

The contribution of ${ }^{40} \mathrm{~K}$ in the ${ }^{238} \mathrm{U}$ and ${ }^{232} \mathrm{Th}$ ROIs were neglected, since the last two were practically unaffected by the ${ }^{40} \mathrm{~K}$ gamma ray spectrum. This assumption was made based on the work done by Rybach [13,14]. It was further assumed that the influence of ${ }^{238} \mathrm{U}$ on the ${ }^{232} \mathrm{Th}$ ROI could also be neglected. By also applying the content characteristics of the standard spectra the calculations could be simplified. The twelve counting efficiencies could then be reduced to six. This then resulted in the following three equations:

The count rates in the ${ }^{40} \mathrm{~K}$ ROI for the potassium, uranium and thorium standards were then

$$
\begin{aligned}
& R_{1,1}=e_{1,1} A_{1,1}+e_{1,2} A_{2,1}+e_{1,3} A_{3,1} \\
& R_{1,2}=e_{1,1} A_{1,2}+e_{1,2} A_{2,2}+e_{1,3} A_{3,2} \\
& R_{1,3}=e_{1,1} A_{1,3}+e_{1,2} A_{2,3}+e_{1,3} A_{3,3}
\end{aligned}
$$

The count rates in the ${ }^{238} \mathrm{U}$ ROI for the uranium and thorium standard were then

$$
R_{2,2}=e_{2,2} A_{2,2}+e_{2,3} A_{3,2}
$$




$$
R_{2,3}=e_{2,2} A_{2,3}+e_{2,3} A_{3,3}
$$

The count rate in the ${ }^{232} \mathrm{Th}$ ROI for the thorium standard were then

$$
R_{3,3}=e_{3,3} A_{3,3}
$$

The solutions for the efficiencies were then as follows:

$$
\begin{aligned}
e_{1,1} & =\frac{R_{1,1}}{A_{1,1}} \\
e_{1,2} & =\frac{R_{1,3} A_{3,2}-R_{1,2} A_{3,3}}{A_{2,3} A_{3,2}-A_{2,2} A_{3,3}} \\
e_{1,3} & =\frac{R_{1,2}}{A_{3,2}}-\frac{A_{2,2}}{A_{3,2}}\left[\frac{R_{1,3} A_{3,2}-R_{1,2} A_{3,3}}{A_{2,3} A_{3,2}-A_{2,2} A_{3,3}}\right] \\
e_{2,2} & =\frac{R_{2,3} A_{3,2}-R_{2,2} A_{3,3}}{A_{2,3} A_{3,2}-A_{2,2} A_{3,3}} \\
e_{2,3} & =\frac{R_{2,2}}{A_{3,2}}-\frac{A_{2,2}}{A_{3,2}}\left[\frac{R_{2,3} A_{3,2}-R_{2,2} A_{3,3}}{A_{2,3} A_{3,2}-A_{2,2} A_{3,3}}\right] \\
e_{3,3} & =\frac{R_{3,3}}{A_{3,3}}
\end{aligned}
$$

Eq. (1) could be used in association with the counting efficiencies to obtain the activity $A_{K}$ (for ROI $1460.8 \mathrm{keV}$ ), $A_{U}$ (for ROI $1764.5 \mathrm{keV}$ ) and $A_{T h}$ (for ROI $2614.5 \mathrm{keV}$ ) related to ${ }^{40} \mathrm{~K},{ }^{238} \mathrm{U}$ and ${ }^{232} \mathrm{Th}$, respectively. The activities in each experimental sample could be extracted from the net count rates $R_{K}, R_{U}$ and $R_{T h}$ that were measured in the three ROIs. Thus, $A_{K}, A_{U}$ and $A_{T h}$ could be obtained by solving the following system of simultaneous equations.

$$
\begin{aligned}
& R_{K}=e_{1,1} A_{K}+e_{1,2} A_{U}+e_{1,3} A_{T h} \\
& R_{U}=e_{2,2} A_{U}+e_{2,3} A_{T h} \\
& R_{T h}=e_{3,3} A_{T h}
\end{aligned}
$$

The activity concentrations of ${ }^{40} \mathrm{~K},{ }^{238} \mathrm{U}$ and ${ }^{232} \mathrm{Th}$ of the various sample points were then calculated and combined in order to be plotted.

\subsection{Study areas and surveys}

The GISPI was used to survey the beaches of Danger Bay and North Bay, close to the town of Saldanha on the West Coast Peninsula of South Africa. The beaches are marked in the image in Figure 1 and the points of each survey are also indicated. 


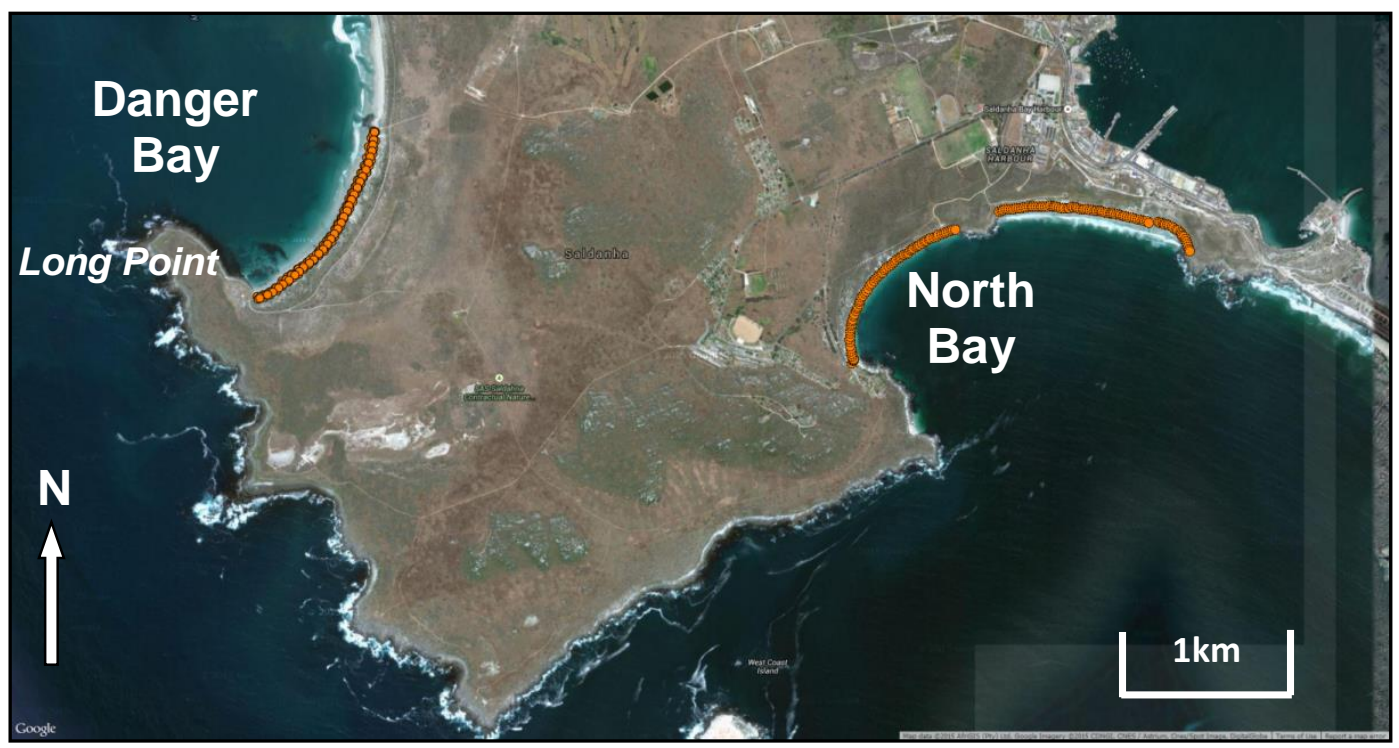

Figure 1: Google Earth image indicating where measurements were conducted on Danger and North Bay beaches. The measuring points are indicated in orange.

The profile of each part of the different beaches were determined and classified as dissipative, intermediate or reflective. Samples of sediment were also taken on these different parts and the particles were sized on the basis of their diameter and then classified by using the Wentworth grading system [15]. The southern part of Danger Bay beach has a highly dissipative profile but slightly changes to an intermediate profile, towards the north. The sediment on the beach varies from medium grain sand to coarse grain sand from the southern part to the northern part, respectively. The beach of southern part of North Bay also demonstrates a highly dissipative profile and changes to intermediate and even reflective towards the northern part. The sediment on North Bay varies from medium grain sand to very coarse grain sand from the south to the north, respectively.

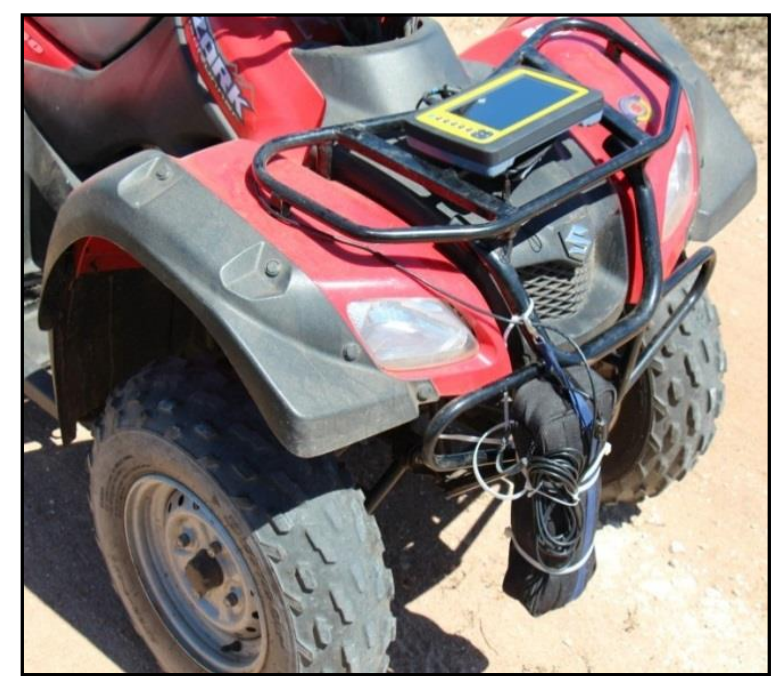

Figure 2: Photographs showing the mounted positions of the GISPI on a quad motorcycle.

The survey of North Bay was conducted on foot while the detector was carried at a height of $20 \mathrm{~cm}$ above the beach sand. The Danger Bay measurements were done by means of a quad motorcycle. The detector was fitted to the front metal structure of the 
motorcycle; also $20 \mathrm{~cm}$ from the ground (see Figure 2). The Yuma rugged table PC was mounted on the front carrier to make it easily accessible to the operator.

All the measured data were plotted and displayed with QGIS software and the results are illustrated in Figures 3, 4, 5 and 7.

\section{Results and Discussion}

The measuring system (GISPI) was initially mounted on a quad motorcycle and in situ measurements were taken by traversing a beach of Danger Bay. The measurements started on the southern part of the beach that are parallel to the incident ocean waves (see the lower part of Figure 3). This part of the beach is sheltered by a granite peninsula, called Long Point, shown in Figure 1. The incoming waves are predominantly incident from a south-westerly direction, which results in fairly low wave energy reaching the southern part of the beach. This Danger Bay beach ends in a rocky granite area, shown in the northern part of Figure 3. The northern part of the beach is more exposed to the incoming waves with subsequent greater wave action and higher wave energy deposits.

No tendency was visible for the sediment of this beach when potassium, uranium or thorium concentrations are overlaid on the map in isolation. However by combining the uranium and thorium in an overlay, a trend became visible. The combining of the uranium and thorium counts were motivated by the fact that both these are heavy elements and that certain chemical similarities also occur. The combination was done by multiplying the radiation counts for the two elements and then overlaying the values on the image of the beach; as illustrated in Figure 3. When these counts were combined, the general pattern revealed an increase from the southern part to the northern part of Danger Bay, coinciding with an increase in wave action across the shore.

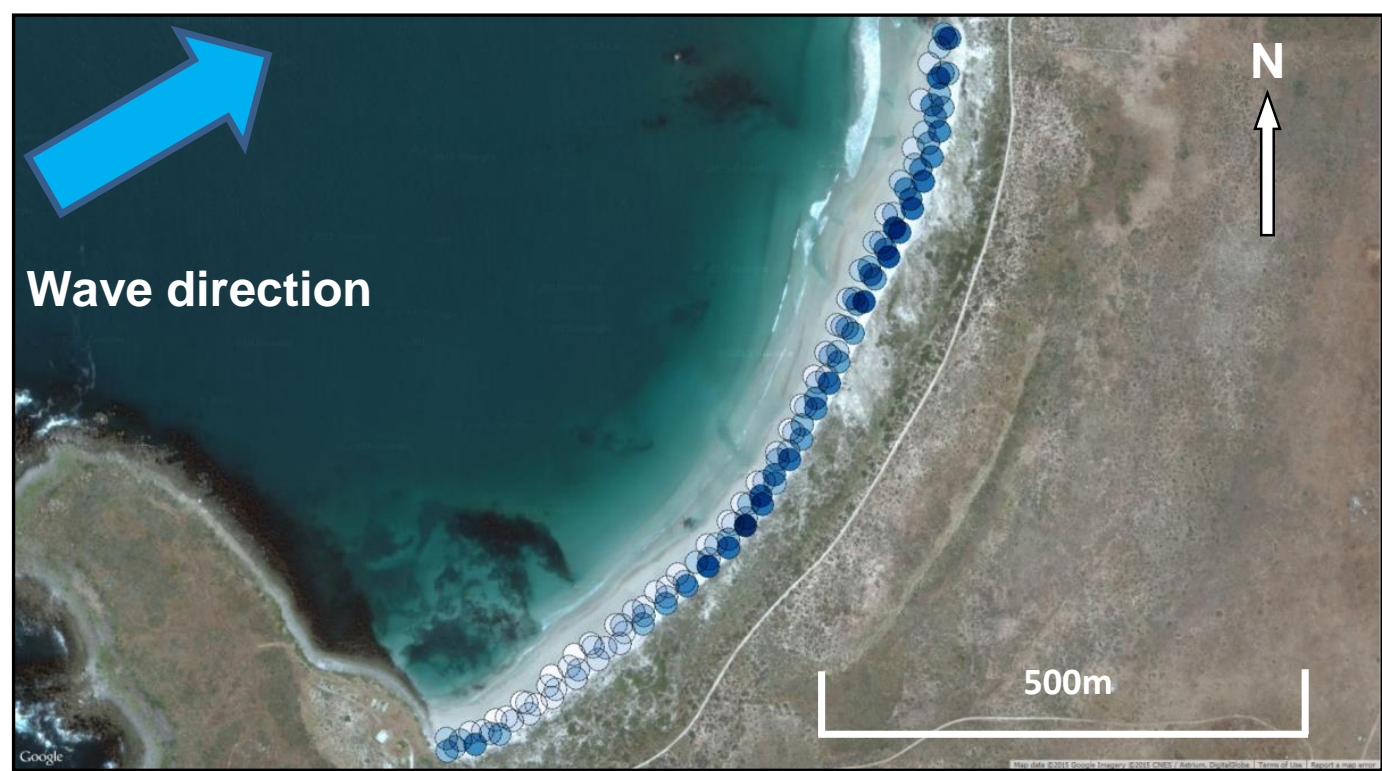

Figure 3: A Google Earth image of a section of Danger Bay with a colour graded overlay indicating a combination of uranium and thorium concentrations in the beach sand. The blue arrow indicates the dominant direction of the incident ocean waves.

The GISPI was carried on foot, when the western beach of North Bay was surveyed. The measurements also started in the southern part of the beach that are parallel to the 
incoming waves. This part is also sheltered by a granite peninsula, visible in Figure 1. The incoming waves in North Bay (west) are predominantly incident from a southerly direction as illustrated on Figure 4 and 5 [16,17]. This results in fairly low wave energy on the southern part of the beach. The sand found in this part of the beach consequently has a small grain size which is consistent with low energy beach deposits. The beach sand mostly consists of finely fragmented ocean sediment and this type of material generally contains low concentrations of natural radionuclides [18].

The beach of North Bay (west) ends at a rocky granite outcrop which swash align with the incoming waves (see Figure 4 and 5). This part of the beach is therefore characterised by higher wave activity. The higher wave activity in this part of the beach consequently deposits sand with a larger grain size, compared to the sand that is deposited at the southern end on the North Bay (west). Figure 4 shows an increase in the potassium concentration along the beach from south to north. The general variation in grain size therefore correlates with the increase in natural potassium, from the southern to the northern part of North Bay (west). There is consequently a relationship between grain size and the potassium concentrations of the beach sediment. The relationship between grain size of sediment and radionuclide concentrations has also been demonstrated in various other studies [19,20].

The very high potassium concentrations in the beach sand of the northern part correspond well with the average potassium concentrations that are found in the surrounding granite [21]. It is therefore likely that the sand in this part originate from regolith of the surrounding granite rocks. Granite rock also contains high levels of uranium and thorium [22]. The uranium and thorium concentrations of North Bay (west) were therefore also combined and plotted. This combined value was overlaid on a Google Earth image of North Bay (west) and is illustrated in Figure 5. It is evident from these results that uranium and thorium follow a similar trend to that of potassium. The combined uranium and thorium values consequently also relate to the grain size.

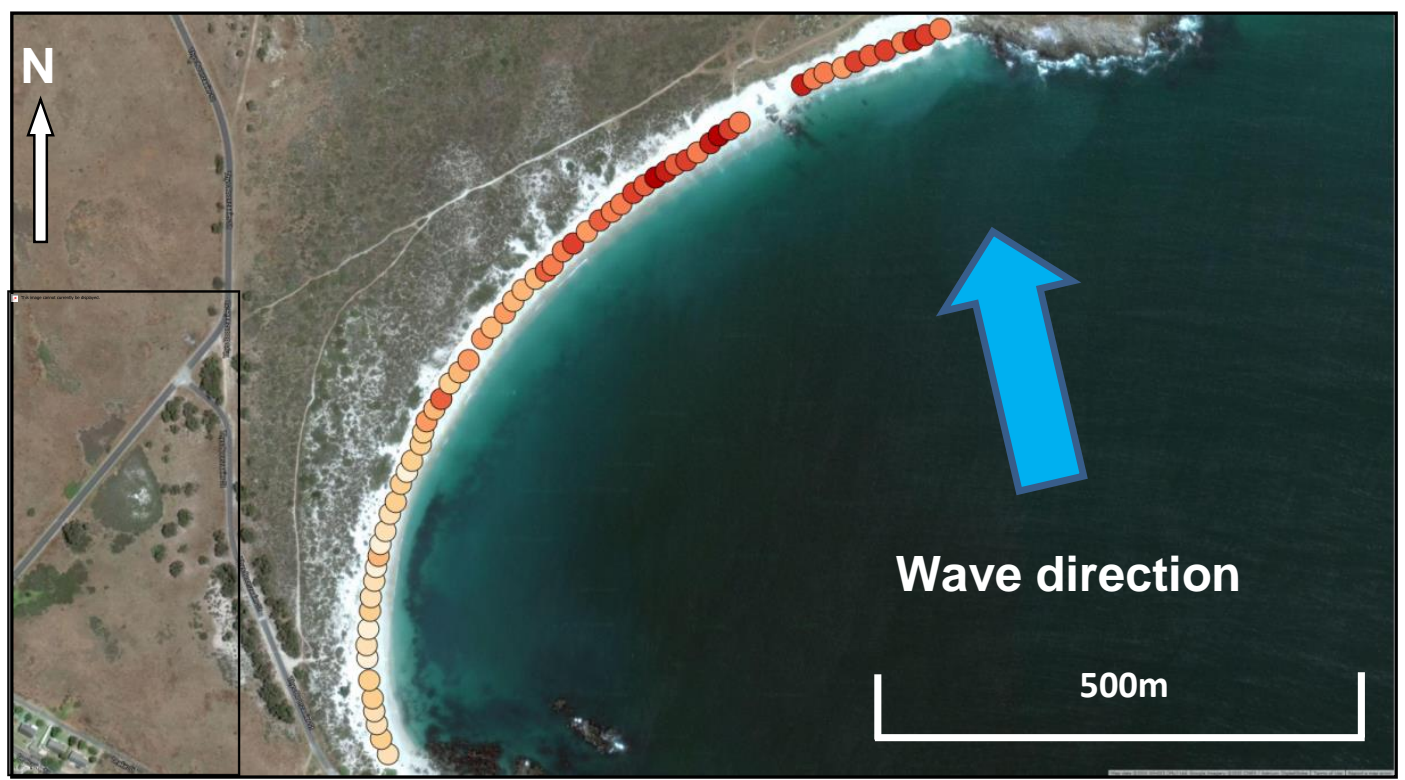

Figure 4: Google Earth image of North Bay (west) with a colour graded overlay indicating the potassium concentrations in the beach sand. The blue arrow indicates the dominant direction of the incident wave approach direction. 


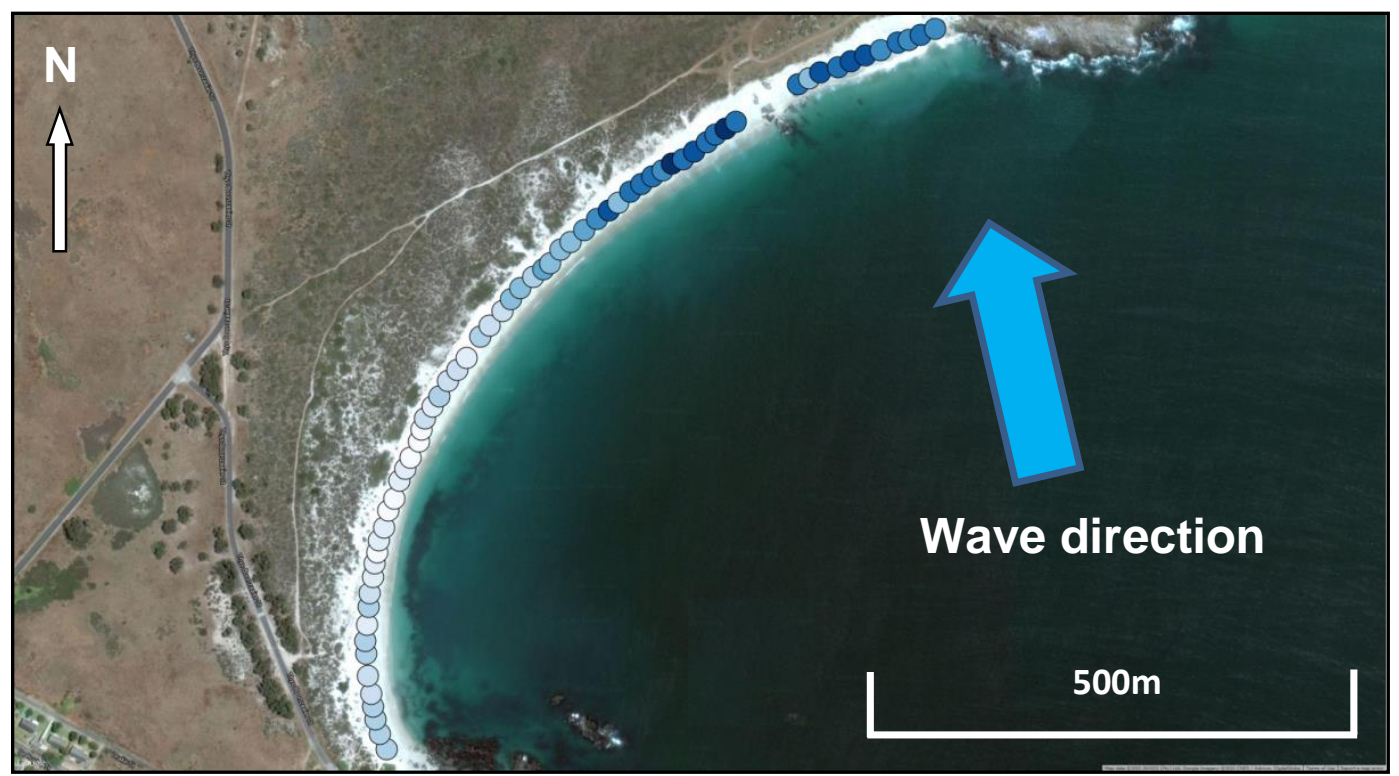

Figure 5: A Google Earth image of North Bay (west) with a colour graded overlay indicating the relative uranium and thorium concentrations in the beach sand. The blue arrow indicates the dominant direction of the incident waves.

The graph in Figure 6 shows the combined uranium and thorium radiation counts as function of the angle between the positions of each measurement and the incident southerly waves. The angle between the positions and the incident ocean waves (wave angle) directly relates to the amount of wave activity; with $0^{\circ}$ position being directly in the wave front and $90^{\circ}$ position being obscured by the granite peninsula. The beach at large angles only receives refracted and diffracted waves with substantial lower energies. The northern part of the beach which is exposed to higher wave energy will consequently have larger grained deposits. The graph in Figure 6 demonstrated an inverse relationship between radionuclide activity and wave angle. The increase in radioactivity relates to larger grained sediments that are deposited by waves with higher energy. These larger grained deposits probably contain regolith of granite rock with intrinsically high levels of radionuclides. 


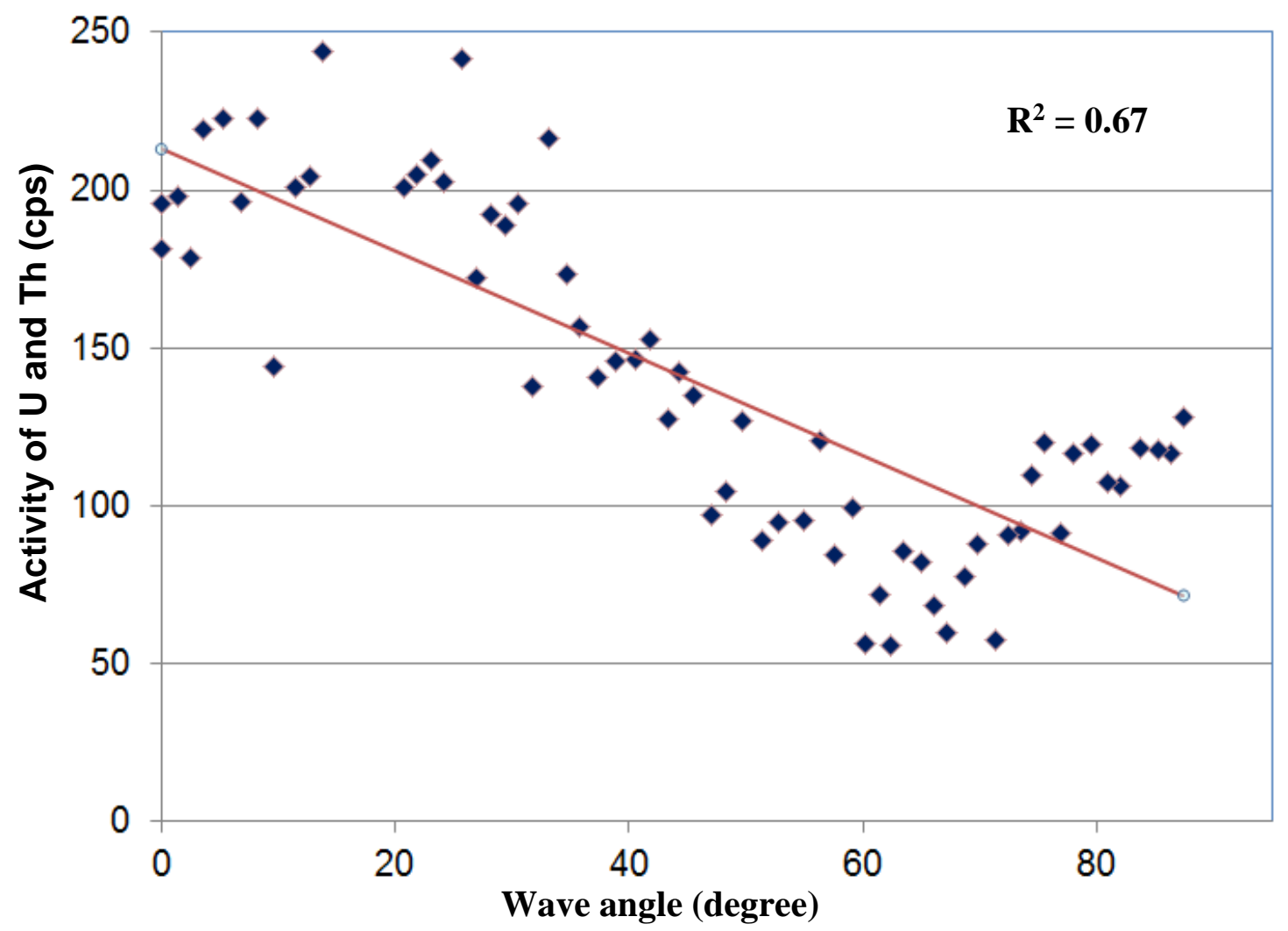

Figure 6: A Graph of the combined uranium and thorium radiation counts as function of the angle between the positions of each measurement and the incident southerly waves in North Bay (west).

The GISPI was also carried on foot, while the eastern beach of North Bay was surveyed. The incoming waves in North Bay (east) are predominantly incident from a southerly direction and the beach also faces directly south, as illustrated in Figure 7. The incident ocean waves traverse directly onto the beach [23,24]. This results in a relatively even level of wave energy along all parts of this beach. The beach sand also does not show any variation in structure or grain size. The measurements conducted along the beach did not show any significant variation in concentrations of potassium, uranium or thorium. This is illustrated in Figure 7, which shows the potassium concentration on North Bay (east).

Figure 8 shows a historic chart of Saldanha Bay that was completed before a breakwater and iron ore jetty were built. The beach of North Bay (west) is marked as A on the chart. The area marked as B is similar in aspect and structure to that of North Bay (west), but is substantially larger. The artificial breakwater and a long iron ore jetty were built during the mid-1970s and are illustrated in red on the chart. These constructions fundamentally changed the wave patterns and the associated sedimentation processes in the bay. 


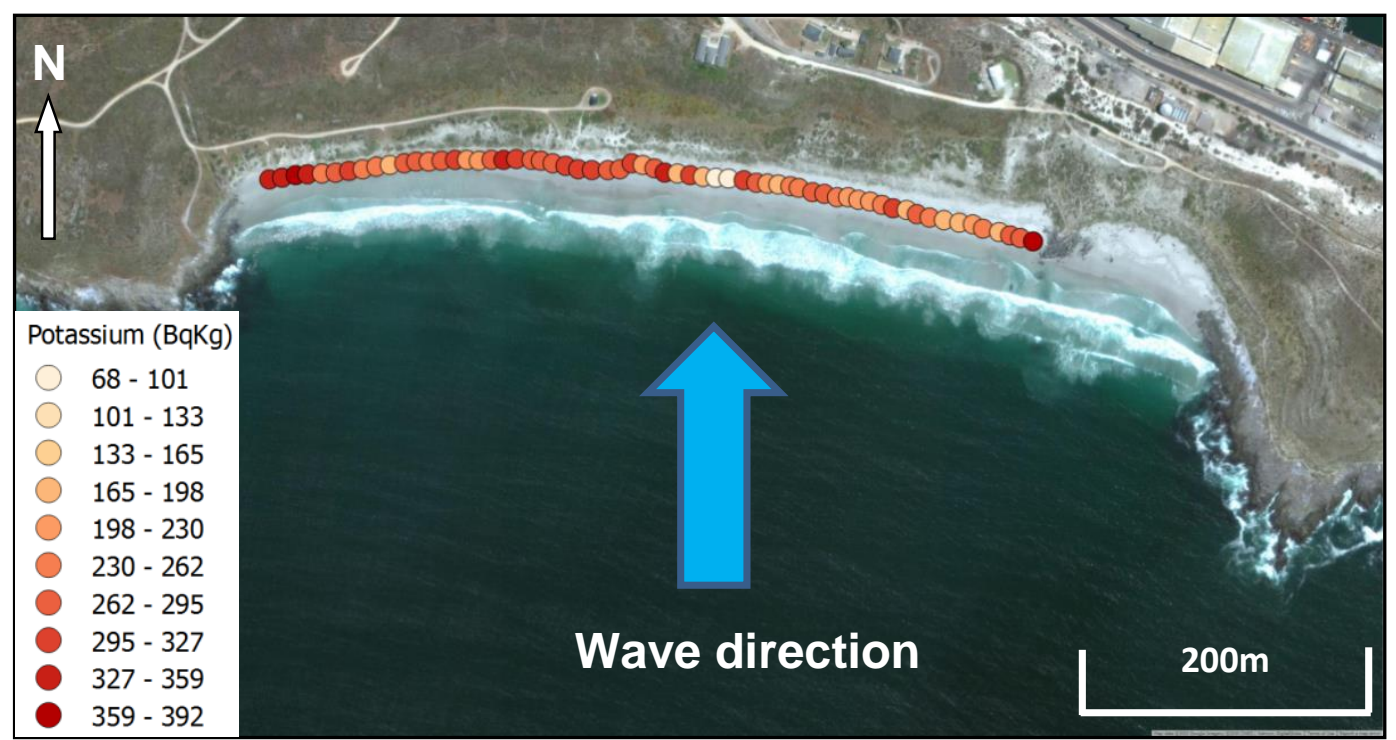

Figure 7: A Google Earth image of North Bay (east) with a colour graded overlay indicating the potassium concentrations in the beach sand. The blue arrow indicates the dominant direction of the incident wave direction.

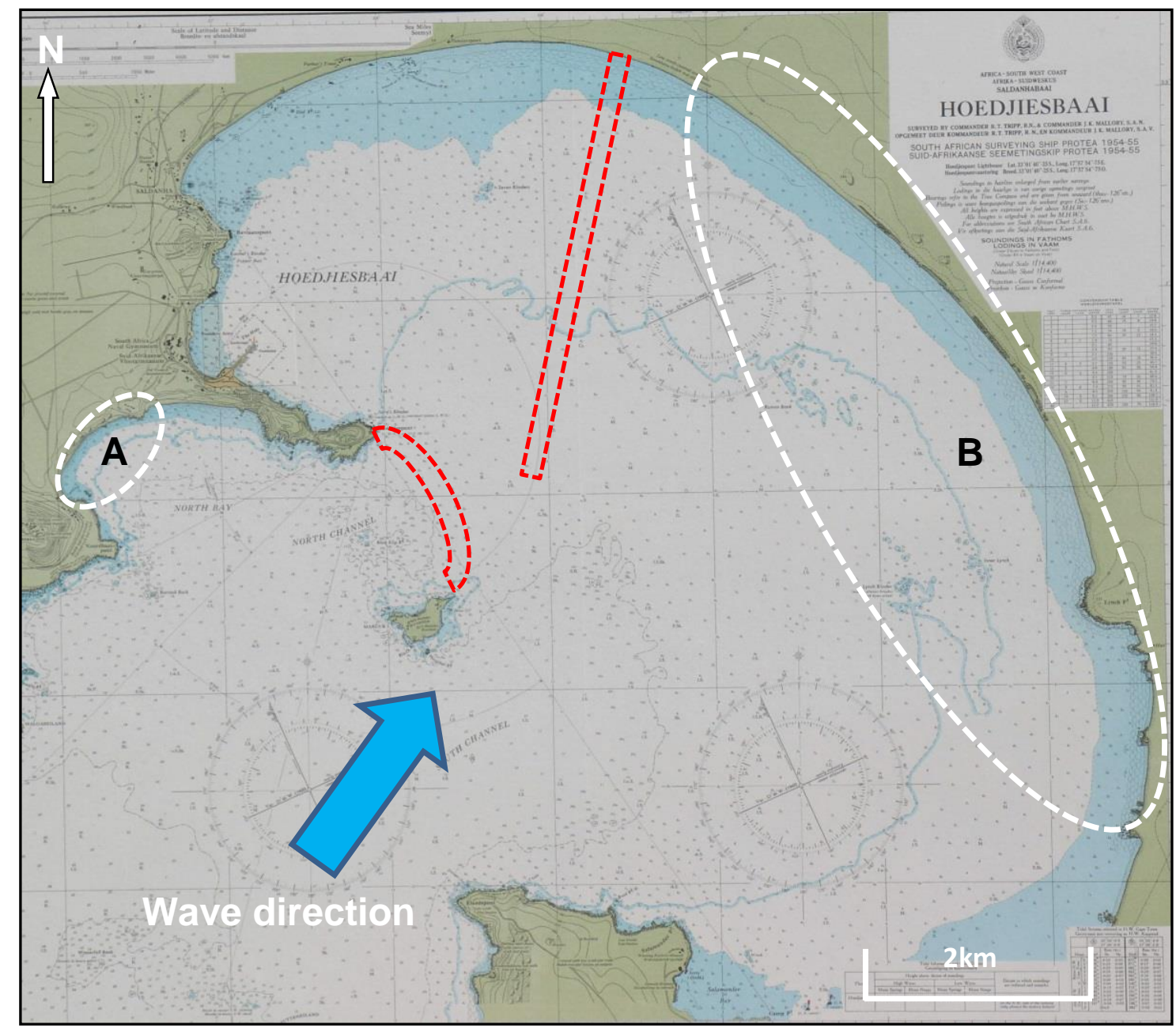

Figure 8: A picture of historic chart of Saldanha Bay which indicated in red where the breakwater and iron ore jetty were built. The areas indicated by A and B is North Bay (west) and the long eastern beach of Saldanha Bay, respectively. 
After the construction of the breakwater and the jetty various beaches in the south of area B started to rapidly erode. These beaches include Paradise Beach, Langebaan Beach and the Leentjiesklip Beach. Drastic measures were taken by the Langebaan Municipality to halt this process which included the construction of a breakwater (See Figure 8) as well as Groyne construction erosion control [25]. Wiese [26] consequently also did a two-dimensional model investigation to determine the factor that were responsible for this rapid erosion. In this work he recommended further study on the influence of wave action on the erosion.

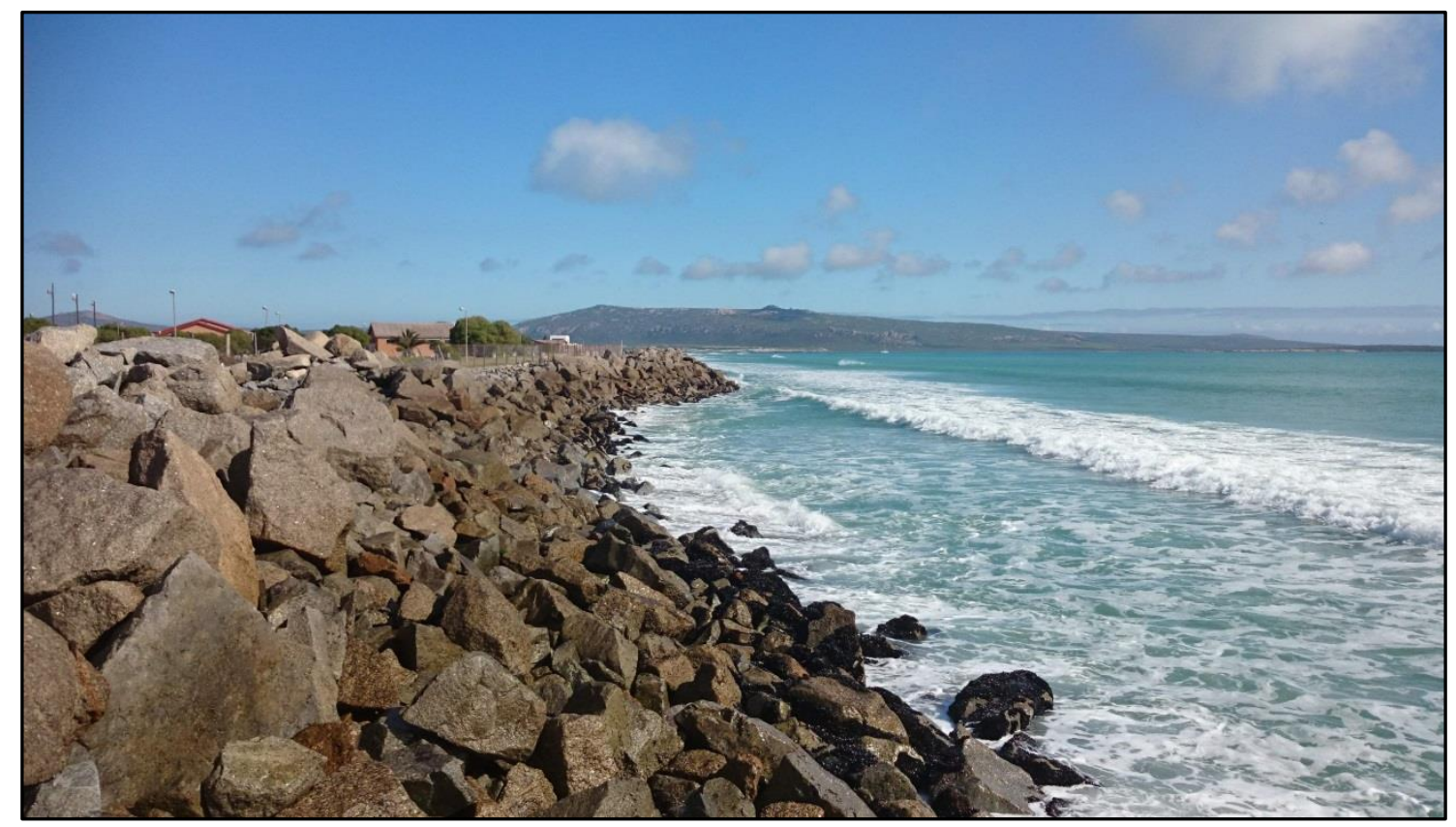

Figure 8: A photograph of a part of the breakwater that was constructed during the 2002 in order to stabilize beaches in and near Langebaan.

Similar fluctuations in wave activity are expected for area B and North Bay (west), due to their physical similarity. If it was therefore assumed that similar sedimentation deposit processes also occurred, before the constructions of the 1970s. Therefore small grained sediment would have gathering in the south on the beaches of area B and larger grained sediment deposits on the beaches in the north. Wave and associated sedimentation patterns inevitably change after the constructions and beach sediment were exposed to new wave activities. The most vulnerable beaches would have been those with fine grained sediment, like the southern beaches in area B. This may be one of the factors that caused the beaches in the southern part of area B to erode over the last three decades.

Results obtained indicate that the relationship between radionuclide concentrations and grain size can provide important information on sediment content and sedimentation processes. The energy of alluvial activity determines the grain size and the type of material that are deposited in sediment. Radiometric surveys can therefore be employed to evaluate the type of material that is deposited, as well as to predict the transportation patterns of alluvial sediment. These radiometric methods can hence assist to better understand coastal sedimentation and beach formation. 


\section{Conclusions}

Various beaches in and around Saldanha Bay were surveyed with an in situ gamma ray instrument (GISPI), either on foot or by quad motorcycle. Potassium, uranium and thorium concentrations where extracted and QGIS software were utilized to overlay the values on Google Earth images. The results show relationships between radionuclide concentrations in the beach sand and wave activity on the beaches. The areas with higher wave activity were also related to the grain size of the beach sediment. This was demonstrated on sections of beaches along Danger Bay and North Bay (west). Both these beaches have areas that are protected from incoming wave by peninsulas. The beach of North Bay (east) which has no barrier, alternatively demonstrated no differences in sand deposited.

This study therefore showed that the origin and the grain size of beach sand can be determined by radiometric surveys, which in turn can assist to better understand beach sedimentation and transportation patterns. These radiometric surveys can be completed in a relatively short time by only traversing the beach. This guarantees that the measurements can be done in situ and at low cost. More radiometric surveys are planned for other areas in Southern African in order to further develop this method and better understanding coastal and beach formation.

\section{Acknowledgements}

The author would like to thank the National Research Fund of South Africa for the funds that they provided for various aspects of this research.

\section{References and Notes}

1. J Bezuidenhout 2012, Mapping of historical human activities in the Saldanha Bay Military Area by using In Situ Gamma Ray Measurements, Scientia Militaria 40. 2. 89-101.

2. Preliminary Assessment of the Marine Environmental Conditions for Liquefied Natural Gas (LNG) Shipment and Transfer Operations for Areas within Saldanha Bay. CSIR Report no. CSIR/BE/HIE/ER/2014/0037/B.

3. S Luger, PMS Monteiro, RC van Ballegooyen, JS Schoonees, and J Moes 1999, Medium term expansion of the Port of Saldanha into Small Bay: A modelling-based predictive study of hydrodynamic, water quality, sediment transport and wave resonance considerations of four alternative port layouts, Volume 1. Text, Volume 2. CSIR Report. ENV-S-C 99050 A, B. Stellenbosch.

4. WG Macdonald, A Rozendaal and RJ de Meijer 1997, Radiometric characteristics of heavy mineral deposits along the west coast of South Africa, Mineralium Deposita 32: 371-381.

5. ER van der Graaf, RL Koomans, J Limburg, and K de Vries 2007, In situ radiometric mapping as a proxy of sediment contamination: Assessment of the underlying geochemical and -physical principles, Applied Radiation and Isotopes 65(5): 619-633.

6. RJ de Meijer 1998, Heavy minerals: from 'Edelstein' to Einstein, Journal of Geochemical Exploration 62 81-103.

7. J Bezuidenhout, The Testing and Implementation of a Transportable and Robust Radioelement Mapping System. Accepted in December 2014 for publication in the South African Journal of Science. 
8. FLIR, 2014. Viewed February 2014, http://gs.flir.com/.

9. Trimble, 2015. Viewed January 2015, http://www.trimble.com/.

10. T McCay, TL Harley, PL Younger, DCW Sanderson and AJ Cresswell 2014, Gamma-ray Spectrometry in Geothermal Exploration: State of the Art Techniques Alistair, Energies, 7(8), 47574780 .

11. P Chiozzi, P De Felice, A Fazio, V Pasquale, and M Verdoy 2000, Laboratory application of $\mathrm{NaI}(\mathrm{Tl}) \gamma$-ray spectrometry to studies of natural radioactivity in geophysics. Applied Radiation and Isotopes 53, 127-132.

12. B Corner, PD Toens, DJ Richards, D Van As, and CM Vleggaar 1979, The Pelindaba facilities for calibrating radiometric field instruments. Publication of Atomic Energy Board, No. PEL-268, Pretoria.

13. L Rybach 1971, Radiometric techniques. In: R E Wainerdi, E A Uken (Eds.). Modern Methods of Geochemical Analysis. Plenum Press, New York.

14. L Rybach 1988, Determination of the heat production rate. In: R Haenel, L Rybach, L Stegena (Eds.). Handbook of Terrestrial Heat-Flow Density Determination, Kluwer Academic Publishers, Dordrecht.

15. CK Wentworth 1922, A scale of grade and class terms for clastic sediments. Journal of Geology, 30, p377-392.

16. Preliminary Assessment of the Marine Environmental Conditions for Liquefied Natural Gas (LNG) Shipment and Transfer Operations for Areas within Saldanha Bay. CSIR Report no. CSIR/BE/HIE/ER/2014/0037/B.

17. S Luger, PMS Monteiro, RC van Ballegooyen, JS Schoonees, and J Moes 1999, Medium term expansion of the Port of Saldanha into Small Bay: A modelling-based predictive study of hydrodynamic, water quality, sediment transport and wave resonance considerations of four alternative port layouts, Volume 1. Text, Volume 2. CSIR Report. ENV-S-C 99050 A, B. Stellenbosch.

18. J Bezuidenhout 2012, Mapping of historical human activities in the Saldanha Bay Military Area by using In Situ Gamma Ray Measurements, Scientia Militaria, 40. 2. 89-101.

19. K Hebinck, H Middelkoop, N van Diepen, ER van der Graaf, and RJ de Meijer 2007, Radiometric fingerprinting of fluvial sediments in the Rhine-Meuse delta, the Netherlands - a feasibility test. Netherlands Journal of Geosciences, Geologie en Mijnbouw 86-3, p229 - 240.

20. RJ de Meijer 1998, Heavy minerals: from 'Edelstein' to Einstein. Journal of Geochemical Exploration 62, 81-103.

21. J Bezuidenhout 2014, Estimation of the background radiation and exposure levels of military personnel in the various military units on the west coast of South Africa. Scientia Militaria, 42, 2.

22. J Bezuidenhout 2014, Estimation of the background radiation and exposure levels of military personnel in the various military units on the west coast of South Africa. Scientia Militaria, 42, 2.

23. Preliminary Assessment of the Marine Environmental Conditions for Liquefied Natural Gas (LNG) Shipment and Transfer Operations for Areas within Saldanha Bay. CSIR Report no. CSIR/BE/HIE/ER/2014/0037/B. 
24. S Luger, PMS Monteiro, RC van Ballegooyen, JS Schoonees, and J Moes 1999, Medium term expansion of the Port of Saldanha into Small Bay: A modelling-based predictive study of hydrodynamic, water quality, sediment transport and wave resonance considerations of four alternative port layouts, Volume 1. Text, Volume 2. CSIR Report. ENV-S-C 99050 A, B. Stellenbosch.

25. Report on a case study by Kaytech engineering fabrics 2011: Groyne construction erosion control Langebaan, Geosynthetics, Ref No: CS ERSN 0356-07/2011.

26. M B Wiese 2013, A two-dimensional mathematical model investigation of the hydrodynamics and sediment transport of Saldanha Bay and Langebaan Lagoon. MScEng Thesis, Stellenbosch University, 2013. 